\title{
NEUROLOGICAL PICTURE
}

\section{Acute longitudinal myelitis as the initial manifestation of Siögren's syndrome}

A 31 year old woman presented with sudden onset of complete paraplegia, decreased sensation for all areas below T7 spinal levels, and urinary retention. Spinal magnetic resonance imaging (MRI) showed multiple and confluent hyperintensities within the entire spinal cord extending into lower part of the medulla oblongata in T2 weighted images (fig 1). Tl weighted, gadolinium enhanced, images also showed multiple confluent contrast enhanced lesions within the entire spinal cord, consistent with longitudinal myelitis (fig 2). Laboratory data and a lip biopsy confirmed the diagnosis of Sjögren's syndrome. As no sicca symptoms were seen in this case, acute longitudinal myelitis was considered to be the initial manifestation of Sjögren's syndrome. Acute disseminated encephalomyelopathy and Devic's disease were deemed unlikely because of the absence of brain and optic nerve lesions. Laboratory data excluded sarcoidosis, a disease of collagen, several infections, and metastatic tumours. Although a few cases have been reported to present acute transverse myelitis as the initial manifestation of Sjögren's syndrome, ${ }^{1-3}$ and two cases with systemic lupus erythematosus showed longitudinal myelitis as its primary manifestation, ${ }^{4}$ there have been, to our knowledge, no reports describing a case of Sjögren's syndrome that initially presented as acute longitudinal myelitis. This case suggests that Sjögren's syndrome could be one of the differential diagnoses of acute longitudinal myelitis.
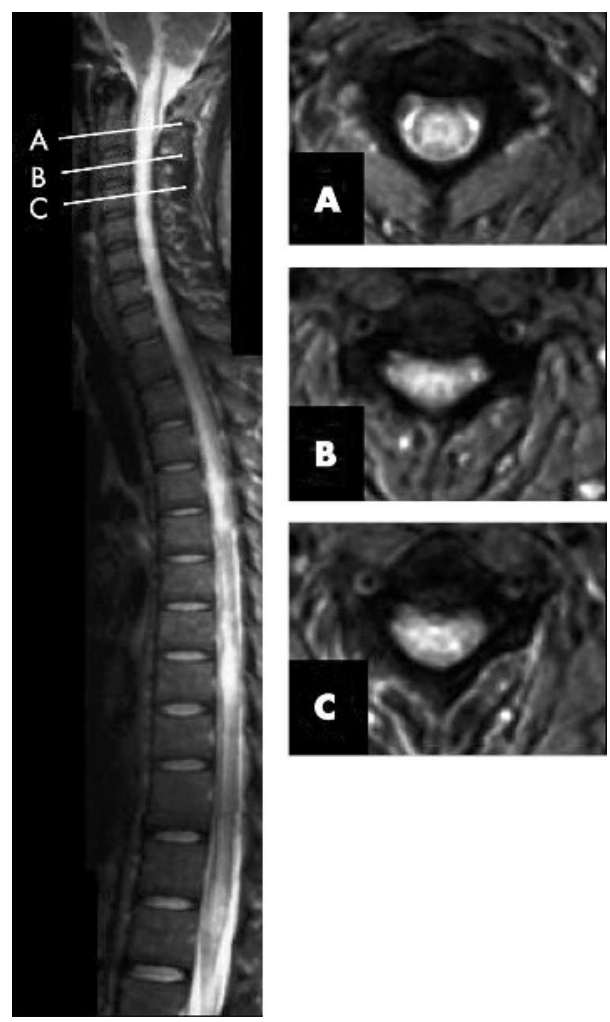

Figure $1 \mathrm{MRI}$ of the entire spinal cord performed on the onset day. T2 weighted sagittal images showed multiple and confluent high signal intensities within the spinal cord, extending from the medulla oblongata to the conus medullaris. The spinal cord shows diffuse swelling. T2 weighted axial images (A-C) showed massive high signal intensities within the cord, especially in the grey matter.

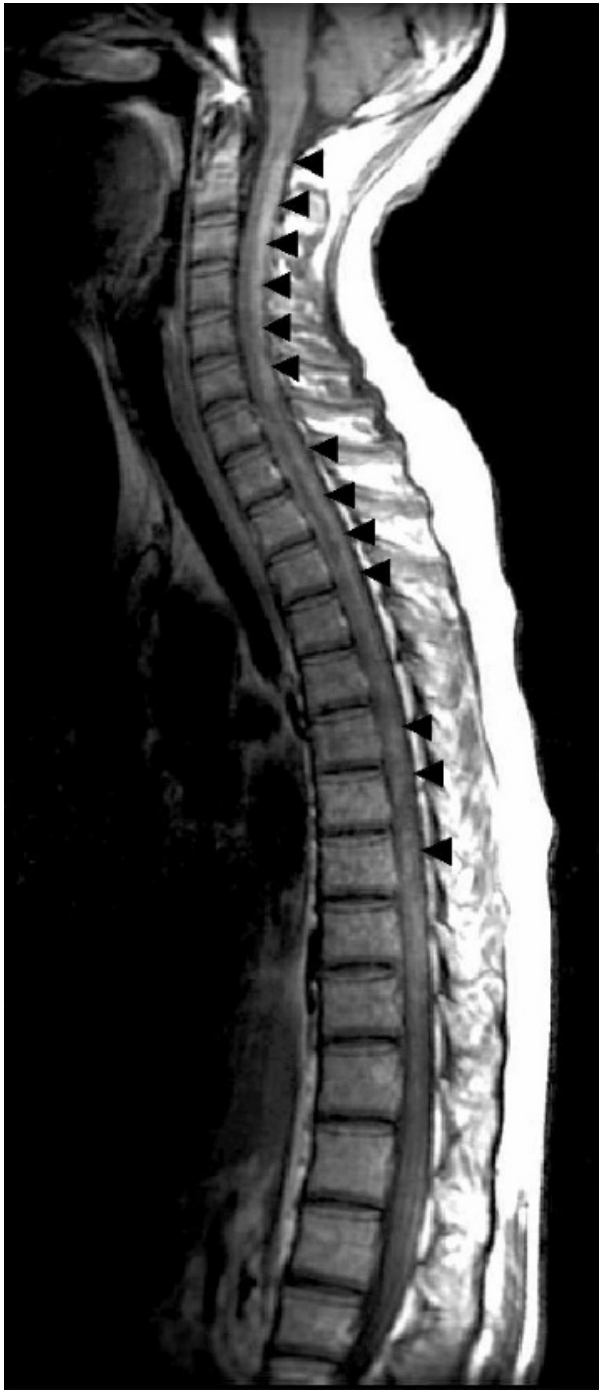

Figure 2. MRI of the entire spinal cord performed a week after the initiation of treatment. T1 weighted, gadolinium enhanced, sagittal images showed multiple and confluent contrast enhanced lesions within the entire spinal cord (arrowheads).

T Yamamoto, S lto, T Hattori Department of Neurology, Graduate School of Medicine, Chiba University, Chiba, Japan, 1-8-1 Inohana, Chuo-ku, Chiba, 260-8670, Japan Correspondence to: Dr S Ito, Department of Neurology, Graduate School of Medicine, Chiba University, 1-8-1 Inohana, Chuo-ku, Chiba, 260-8670, Japan; sito@faculty.chiba-u.jp

Competing interests: None declared.

\section{References}

1 Manabe $Y$, Sasaki $C$, Warita $H$, et al. Siögren's syndrome with acute transverse myelopathy as the initial manifestation. J Neurol Sci 2000;176:158-61

2 Yanagihara C, Nakaji K, Nishimura Y. A case of primary Sjögren's syndrome with acute transverse myelopathy and polyneuropathy as the initial manifestations. Rinsho Shinkeigaku 2001;41:50-5.

3 Arai C, Furutani R, Ushiyama M. A case of Sjögren's syndrome with subacute transverse myelitis as the initial manifestation. Rinsho Shinkeigaku 2002;42:613-18.

4 Chen HC, Lai JH, Juan CJ, et al. Longitudinal myelitis as an initial manifestation of systemic lupus erythematosus. Am J Med Sci 2004;327:105-8.

5 Lehnhardt FG, Impekoven P, Rubbert A, et al. Recurrent longitudinal myelitis as primary manifestation of SLE. Neurology 2004;63:1976. 